\title{
PRODUÇÃO DO CUIDADO EM SAÚDE MENTAL: PRÁTICAS TERRITORIAIS NA REDE PSICOSSOCIAL
}

\author{
PRODUCTION OF CARE IN MENTAL HEALTH: TERRITORIAL \\ PRACTICES IN THE PSYCHOSOCIAL NETWORK
}

\author{
Daniella Barbosa Campos ${ }^{1}$ \\ (D) [0000-0002-8796-8510], Indara Cavalcante Bezerra² \\ Maria Salete Bessa Jorge ${ }^{1}$ \\ 1 Universidade Estadual do Ceará, Departamento de Ciências e Saúde, Fortaleza, Ceará, Brasil. \\ <dane_barbosa@hotmail.com> \\ ${ }^{2}$ Universidade de Fortaleza, Departamento de Ciências e Saúde, Fortaleza, Ceará, Brasil.
} [0000-0003-0647-2490]

\begin{abstract}
Resumo Trata-se de um estudo qualitativo que analisou práticas de cuidado territoriais em saúde mental realizadas por enfermeiros, agentes comunitários de saúde e usuários dos centros de atenção psicossocial e da atenção básica. Foram realizadas entrevistas, grupos focais e observação livre com 60 participantes de Fortaleza, Ceará, em 2017. A análise das informações fundamentou-se na hermenêutica-dialética ante um exercício interpretativo crítico e reflexivo. Os resultados evidenciaram que o processo de territorialização é realizado pela equipe multidisciplinar da Estratégia Saúde da Família, sem a participação dos profissionais do centro de atenção psicossocial. Embora estes, por vezes, realizem práticas comunitárias, persiste a valorização de ações dentro do próprio serviço e na medicalização do sofrimento psíquico, sem considerar as singularidades dos sujeitos e sem articulação com os serviços da atenção básica. Os agentes comunitários de saúde, se treinados, são atores potencialmente estratégicos para atuar na interface da saúde mental com a atenção básica. Com efeito, o panorama da saúde mental urge pela transformação de um modelo que privilegie a reflexão de novas ações em múltiplas dimensões, com ênfase na articulação dos serviços e na capacitação dos trabalhadores que atuam nesse âmbito.
\end{abstract}

Palavras-chave assistência à saúde; atenção primária à saúde; saúde mental; serviços de saúde mental; agentes comunitários de saúde.
Abstract This is a qualitative study that analyzed the practices of territorial care in mental health developed by nurses, community health workers and users of the psychosocial care centers and of primary health care. We conducted interviews, focus groups and free observation with 60 participants from the city of Fortaleza, state of Ceará, Brazil, in 2017. The data analysis was based on hermeneutics-dialectics in the face of a critical and reflective interpretive exercise. The results revealed that the territorialization process is developed by the multidisciplinary team from the Health of the Family Strategy, without the participation of the professional of the psychosocial care center. Even though they may sometimes develop community practices, they still privilege actions within the service itself and those focusing on the medicalization of mental suffering, without taking into consideration the singularities of the subjects, and with no interaction with the primary health care services. The community health workers, if trained, are potentially strategic actors to work on the interface between mental health and primary health care. In fact, the mental health outlook urges for the transformation of a model that privileges the reflection of new actions into multiple dimensions, with an emphasis on the connection of the services and the training of the workers that act in this context.

Keywords health care; primary health care; mental health; mental health services; community health workers. 


\section{Introdução}

O cuidado em saúde mental no contexto da atenção primária à saúde (APS) e dos centros de atenção psicossocial (CAPS) deve ser planejado por meio de estratégiasterritoriais baseadasnasnecessidades dosusuários.Dessaforma, promove-seumaassistênciaeficienteedequalidadequepossibiliteproduzir saúde nos espaços de vida cotidiana, com reinserção do usuário ao convívio social (Brasil, 2011; 2013). Este modo de cuidar depende de profissionaisqualificados, capazes de refletir sobreos processos detrabalhoanteuma perspectivacoletivaebiopsicossocial,voltadapararesponderdinamicamenteàs demandas das pessoas em sofrimento psíquico (Marcolino, Fantinattie Gozzi, 2018).

No que se refere às práticas e processos constituintes desses serviços, persistem desafios complexos, que vão desde a formação dos trabalhadores que atuam no âmbito da saúde mental e em suas interfaces, como educação, assistência social e outros setores, até a articulação entre os serviços e a valorização de práticas comunitárias permanentes que envolvam as potencialidades do território (Marcolino, Fantinatti e Gozzi, 2018; Moura e Silva, 2015; Castro e Maxta, 2010; Onocko-Campos et al., 2009).

Nessa perspectiva, Prata et al. (2017) afirmam que o conceito de 'território' fundamenta-se numa compreensão do 'ser' indissociável do contexto em que vive, do lugar de pertença, no qual se constroem as relações decorrentes de situações históricas, ambientais e sociais. Segundo os referidos autores, os territórios são vivos e constituem-se pela experiência das pessoas que neles convivem, por meio da história, da cultura, dos movimentos sociais e das ações políticas (Prata et al., 2017).

No tocante à organização dos serviços de saúde, para que operem segundo a lógica do território, devem-se buscar e ativar os recursos locais existentes no entorno da comunidade. Desse modo, são bem-vindas parcerias com organizações, instituições ou grupos de pessoas envolvidas com arte ou projetos sociais potencializadores que envolvam educação, esporte, trabalho, entre outros, no sentido de reconhecer as singularidades dos sujeitos e estimular a participação social (Lima e Yasui, 2014).

A literatura internacional evidencia lacunas no acesso e na prestação de serviços de saúde mental em todo o mundo e aponta, dentre outras estratégias, que práticas territoriais de cuidado em saúde mental têm o potencial de ofertar uma maior inserção e aceitação dos usuários na comunidade.

Nos Estados Unidos, apenas um terço das pessoas que precisam recebem cuidados de saúde mental adequados (Kilbourne et al., 2018). No Peru, estudo recente expressa que os transtornos mentais afetam uma em cada cinco pessoas, representando a principal causa da carga de doenças e que, apesar da alta prevalência e impacto negativo na vida dos pacientes, esses transtornos são amplamente subdiagnosticados e subtratados (Diez-Canseco et al., 2018). Em 
um estudo realizado na Argentina, foi constatado que pessoas com transtornos mentais que convivem em meio à comunidade promovem a aceitação de outras pessoas, o que facilita a socialização e o cuidado à saúde (Mulyale et al., 2014). Em outro estudo, produzido no Canadá, verificou-se que os cuidados territoriais tornaram-se uma necessidade dos usuários, que elaboraram uma estratégia de cuidado em saúde mental (Brasil, 2012).

A Reforma Psiquiátrica teve as suas primeiras experiências exitosas na França e Itália. Na França, surgiram as primeiras propostas de modernização dos hospitais psiquiátricos, para reduzir as práticas violentas. Na Itália, o foco era o combate ao manicômio e pela revisão do estatuto jurídico do doente mental, por entender que esses dois aspectos estavam intimamente relacionados (Maciel, 2010).

No Brasil, as práticas em saúde mental foram constituídas e impulsionadas pela Reforma Psiquiátrica em meio a lutas pela redemocratização do país, com base no Movimento dos Trabalhadores de Saúde Mental (MTSM), no final dos anos 1970. Tal movimento abriu espaço para intensas discussões acerca do modelo manicomial, o qual era centrado na exclusão de usuários em sofrimento psíquico, representando uma cultura autoritária, baseada na violência contra os que não se enquadram nos modelos sociais ideais (Arruda, 2011; Lancetti e Amarante, 2006).

A Reforma Psiquiátrica no Brasil impulsionou as lutas pelo reconhecimento da saúde mental como um campo de atuação com perspectivas plurais, complexas, intersetoriais e inclusivas da transversalidade de saberes (Nunes, Guimarães e Sampaio, 2016). Ademais, este movimento resultou em um novo modelo de atenção psicossocial territorial, caracterizado pela ampliação do conceito de saúde, que passou a considerar as concepções do cuidado integral e humanizado, com objetivo de superar a visão biomédica e individualizada da saúde (Souza e Afonso, 2015). Tal perspectiva condicionava o seu significado à ausência de doença ou à utopia de completo bem-estar biopsicossocial.

O novo modelo de saúde mental abrange, como eixo principal, a reestruturação da assistência, caracterizada pela desospitalização de pacientes internados por longos períodos e a criação de serviços substitutivos, cujos dispositivos possam lidar com o sofrimento mental $\nabla$ dentre eles, o CAPS (Onocko-Campos et al., 2009).

Nesse sentido, os CAPSs assumiram caráter estratégico na reorientação do modelo de atenção à saúde mental, com objetivo de garantir a universalidade, equidade, integralidade, descentralização, participação social e democrática dos diferentes atores sociais e resolubilidade do cuidado assumindo posição central na articulação entre os serviços de saúde (Souza e Afonso, 2015; Brasil, 2011). Para tanto, instituiu-se a Rede de Atenção Psicossocial (RAPS), cujo objetivo geral é o de ampliar o acesso à atenção psicossocial da população em geral e garantir a articulação e integração dos pontos de atenção das redes 
de saúde no território, qualificando o cuidado por meio do acolhimento, do acompanhamento contínuo e da atenção às urgências (Ardila-Gómez et al., 2015; Brasil, 2011).

Para uma maior articulação entre os serviços, foi criada a RAPS, que surgiu como proposta organizativa dos serviços de saúde mental, instituída pela Portaria Ministerial n³.088, em 2011, cuja finalidade é a criação, ampliação e articulação de pontos de atenção à saúde para pessoas com sofrimento ou transtorno mental e com necessidades decorrentes do uso de crack, álcool e outras drogas, no âmbito do Sistema Único de Saúde (SUS).

Embora existam políticas nacionais que sustentem os direitos das pessoas com transtornos mentais (Brasil, 2013; 2011), persistem desafios para a implementação dessas políticas e para a efetividade das práticas que garantam esses direitos, arduamente conquistados. Estudo recente de revisão integrativa da literatura científica nacional evidenciou as dificuldades que permeiam as práticas de cuidado em saúde mental na atenção básica oferecidas ao usuário em sofrimento psíquico, dentre as quais: fragilidade na formação e capacitação profissional; modelo biomédico medicalizante e excludente; falhas no encaminhamento e fragmentação do cuidado por especialidade; ausência de rede de apoio à família; ausência de práticas na comunidade que potencializem o cuidado da atenção básica e psicossocial no território (Fernandes, Matsukura e Lourenço, 2018).

Por outro lado, o apoio matricial representa uma estratégia integrativa na interface da atenção psicossocial com a atenção básica, pois propõe a superação da lógica vertical de conhecimentos, buscando a interação entre equipes, somada à subversão da lógica de encaminhamentos, referências e contrarreferências. Trata-se de um apoio teórico-pedagógico voltado para a capacitação de uma equipe profissional, com base no trabalho e orientação em conjunto com uma equipe de referência (Bezerra e Jorge, 2018). Para exemplificar, a equipe do CAPS pode oferecer apoio matricial às equipes da Estratégia Saúde da Família (ESF) no tocante ao cuidado em saúde mental.

Outros estudos nacionais brasileiros corroboram que o cuidado em saúde mental desenvolvido na comunidade territorial promove um maior vínculo dos usuários e familiares com os serviços, pois, nesses espaços coletivos, é possível acessar a diversidade de demandas dos usuários e oferecer um cuidado específico às necessidades de cada indivíduo (Teixeira, Couto e Delgado, 2015; Souza e Carvalho, 2014).

Com base no exposto, a realização desta pesquisa é ponto de partida para compreender quais as práticas de cuidado realizadas no território são exercidas pelo centro de atenção psicossocial CAPS e pela APS, com destaque nos arranjos organizativos desses serviços. Pretende-se, ainda, identificar as lacunas e potencialidades existentes no campo empírico, com vistas a subsidiar 
reflexões para auxiliar a tomada de decisão dos gestores, além de qualificar a oferta terapêutica, ensejando maior resolubilidade.

A análise possibilitará uma compreensão das práticas exercidas extramuros pelos serviços com bases territoriais e permitirá a formulação e proposição de estratégias que propiciem qualificar a atenção às pessoas em sofrimento psíquico. Do mesmo modo, tal análise poderá sensibilizar os profissionais de saúde a produzirem a longitudinalidade do cuidado com vistas a alcançar a integralidade da assistência, por meio da formação de vínculos, confiança, empatia e corresponsabilização. Com este conhecimento, será possível organizar um cuidado baseado nas necessidades singulares de cada indivíduo.

Diante dos aspectos teórico-conceituais apresentados, questiona-se quais são as práticas de cuidado exercidas pelos profissionais do CAPS e APS no território adscrito. Com efeito, neste artigo, objetivou-se analisar as práticas de cuidado territoriais em saúde mental realizadas pelos profissionais do CAPS e da APS.

\section{Métodos}

Trata-se de uma pesquisa qualitativa ante uma perspectiva crítica e reflexiva fundamentadanahermenêutica-dialética, aqual possibilitouoentendimento do fenômeno social, cujo objeto refere-se às práticas de cuidado territoriais em saúde mental e suas relações no campo da saúde (Minayo, 2010).

Para Minayo (2010), esse tipo de estudo contempla um universo de significados, motivos, crenças, valores e atitudes que correspondem a um espaço mais profundo das relações, dos processos e dos fenômenos que não podem ser restringidos às mensurações de variáveis. Além de possibilitar desvelar processos sociais ainda pouco conhecidos, permite a construção de novas abordagens e formação de conceitos durante a investigação.

Nesse sentido, o referencial metodológico baseou-se na composição metodológica da hermenêutica e dialética, buscando a síntese dos processos compreensivos e críticos, ao considerar que a mesma razão que compreende, esclarece e reúne, também contesta, dissocia e critica (Minayo, 2010; 1999).

O estudo foi desenvolvido no município de Fortaleza, Ceará, que possui 119 bairros organizados, administrativamente, em seis Coordenações Regionais em Saúde (CORES - I, II, III, IV, V e VI). Cada regional possui uma rede de equipamentos de saúde para atendimento da população mais próximo da sua residência (Fortaleza, 2017).

A rede é composta de unidades de atenção primária à saúde (UAPSs), hospitais de atenção secundária e terciária, unidades de pronto-atendimento (UPAs), CAPSs infantil, geral e álcool e drogas (AD), centros de especialidades odontológicas (CEOs), centrais de distribuição de medicamentos no terminal, 
equipes de consultório na rua, Espaço Ekobé, além de espaços para desenvolvimento de práticas integrativas e complementares à saúde, que, frequentemente, funcionam dentro das unidades de APS (Fortaleza, 2017).

A Tabela 1 expressa a distribuição desses equipamentos de saúde nas CORESs, referentes aos serviços de saúde mental e atenção básica.

Tabela 1.

\begin{tabular}{|c|c|c|c|}
\hline \multicolumn{4}{|c|}{ Distribuição dos Equipamentos de Saúde por Regional em Fortaleza } \\
\hline CORES & $\begin{array}{l}\text { Equipamentos } \\
\text { de Saúde }\end{array}$ & Saúde Mental & UAPS \\
\hline 1 & 23 & $\begin{array}{l}1 \text { CAPS Geral, } 1 \text { CAPS AD, } 1 \text { Unidade de Acolhimento, } 1 \text { serviço de residência } \\
\text { terapêutica }\end{array}$ & 14 \\
\hline II & 18 & $\begin{array}{l}1 \text { CAPS Geral, } 1 \text { CAPS AD, } 1 \text { Unidade de Acolhimento, } 1 \text { serviço de residência } \\
\text { terapêutica }\end{array}$ & 12 \\
\hline III & 23 & 1 CAPS Geral, 1 CAPS AD, 1 CAPS Infantil & 18 \\
\hline IV & 20 & 1 CAPS Geral, 1 CAPS AD & 13 \\
\hline $\mathrm{V}$ & 30 & 1 CAPS Geral, 1 CAPS AD, 1 serviço de residência terapêutica & 24 \\
\hline VI & 36 & 1 CAPS Geral, 1 CAPS AD, 1 CAPS Infantil, 1 Unidade de Acolhimento & 26 \\
\hline
\end{tabular}

A escolha para este estudo delimitou a participação de seis unidades de atenção primária à saúde e seis centros de CAPSs de todas as CORESs de Fortaleza.

Desse modo, dentre as 117 UAPSs existentes em Fortaleza, foram selecionadas seis, mediante sorteio, uma em cada CORES, além da inclusão de todos os CAPSs Gerais do município, ou seja, os seis CAPSs Gerais.

Participaram do estudo 60 pessoas, dentre as quais 24 enfermeiros, sendo 12 vinculados às UAPSs (dois de cada UAPS) e 12 vinculados aos CAPSs gerais (dois de cada CAPS); 12 agentes comunitários de saúde (ACS) e 24 usuários (dois de cada UAPS e dois de cada CAPS). A participação dos enfermeiros foi vinculada aos critérios de seleção pré-estabelecidos, a saber: trabalhadores de saúde precisam possuir, no mínimo, um (1) ano de trabalho na ESF ou CAPS; usuários devem ser assistidos na rede de atenção primária e psicossocial e atendidos no CAPS geral. Os critérios de exclusão foram: trabalhadores de saúde que se encontrassem ausentes por férias ou licença no período da coleta de dados; usuários que possuíssem quadro clínico que invalidasse a sua participação na pesquisa, como intensa agitação ou sob sedação, por exemplo.

No que se refere à coleta e organização dos dados, a pesquisa em campo ocorreu no período de abril a maio de 2017, por meio de entrevistas semiestruturadas com profissionais e usuários, grupo focal com os ACS, além da observação sistemática livre para a aproximação e reconhecimento do lócus empírico.

A coleta das informações ocorreu em dois momentos. No primeiro, realizaram-se visitas aos equipamentos de saúde (UAPSs e CAPSs). Na unidade 
de saúde, fez-se uma observação livre para obter uma melhor compreensão do contexto da pesquisa; não houve teste-piloto.

No segundo momento, ocorreram as entrevistas com os enfermeiros e usuários nas UAPSs e nos CAPSs gerais, ambos equipamentos de APS. As entrevistas foram feitas em salas reservadas para evitar a interferência de outras pessoas e respeitar a privacidade dos participantes.

Por fim, visando compreender a complexidade do objeto de estudo proposto, foi realizado um grupo focal com os ACS em uma das UAPSs selecionadas para o estudo, a fim de complementar ou confrontar as informações. Foram disparados os seguintes questionamentos: Quais as práticas de cuidado em saúde mental são realizadas no território? Como se dá a atuação e articulação dos profissionais dos CAPSs e da atenção básica na prática do cuidado territorial?

Para a análise, organização e sistematização do material coletado, usamos o fluxograma analisador proposto por Assis e Jorge (2010), o qual recomenda as três etapas propostas por Minayo (2008) e retraduzidas pelas referidas autoras: ordenação, classificação e análise final dos dados. Tais fases são interrelacionadas, dinâmicas e complementares. A ordenação envolve a transcrição de entrevistas, leitura do material empírico e organização dos dados que emergem do texto em diferentes conjuntos de sentidos. A classificação dos dados permite a construção, o agrupamento dos conjuntos de sentidos em temas dos dados empíricos e, por fim, a análise final enfatiza a interpretação, possibilitando estabelecer as complementaridades, as convergências, as divergências e o inusitado, oriundos do material empírico apreendido.

A análise final seguiu a hermêutica-dialética orientada por Minayo (1999; 2010) e Assis e Jorge (2010), na qual foi possível estabelecer as complementaridades, as convergências, as divergências e o inusitado do material empírico apreendido.

Os aspectos éticos foram respeitados, de acordo com a Resolução nº 466/2012 do Conselho Nacional de Saúde/Ministério da Saúde, que aborda as pesquisas envolvendo seres humanos. O comitê de ética, com protocolo $n^{\circ}$ 2.005.057 (Brasil, 2012), aprovou a pesquisa. Os profissionais que concordaram em participar do estudo assinaram o termo de consentimento livre e esclarecido, que assegura o anonimato dos participantes, autoriza a utilização e a publicação dos dados obtidos, e além disso, esclarece os objetivos da pesquisa, a justificativa e a garantia do sigilo das informações, ratificando o compromisso ético com os interlocutores. Neste artigo, os enfermeiros estão identificados pela abreviação 'Enf.', seguida da ordem das entrevistas (de 1 a 24); os ACS, pela sigla 'ACS-GF', pois as falas foram originadas no grupo focal, e os usuários, pela própria palavra, seguida de 1 a 24, também pela ordem das entrevistas. 


\section{Resultados e Discussão}

A Reforma Psiquiátrica consolidou o modelo de atenção psicossocial territorial, com práticas voltadas para o território onde os usuários em sofrimento psíquico vivem e se relacionam (Lancetti e Amarante, 2006). Estes lugares são capazes de revelar as singularidades, diversidades e realidades, concepções essenciais paraocuidadonocampo psicossocial.Os discursos dos trabalhadoresprofissionais de saúdeeusuários possibilitaram compreenderosavançose desafiosnaorganizaçãodosserviçosaotraduziremarealidadevivenciadapara consolidar um novo modelo de atenção psicossocial territorial local.

O território é um importante lócus de cuidado em saúde mental e um poderoso componente para análise e planejamento das ações em saúde. A dimensão que o espaço recebe para contemplar as questões relacionais, socioculturais e políticas daquela sociedade vai configurando outro território, ou seja, marcando-o como um território dinâmico, ativo e polivalente (Silva e Pinho, 2015).

Sabe-se que, no modelo da atenção psicossocial territorial, o objeto de intervenção se desloca da doença para o sujeito em sofrimento psíquico. As práticas devem sair do escopo medicamentoso preponderante para visualizar o usuário como participante principal no tratamento. Este processo envolve a família e, eventualmente, esferas comunitárias e suas respectivas constituições política, histórica e sociocultural, reconhecendo-se a complexidade do processo saúde-doença. Desse modo, amplia-se a visão terapêutica da remoção de sintomas para a adaptação à realidade e à supressão de carências socioeconômicas e culturais. O cuidado busca envolver o diálogo e o acompanhamento longitudinal da pessoa, reconhecendo o território como novo cenário das ações em saúde mental (Castro e Maxta, 2010; Guimarães, 2012; Sampaio, Guimarães e Abreu, 2010).

Desvelar o território é uma das atribuições dos profissionais de saúde da RAPS, com uso da ferramenta chamada territorialização, que permite ao profissional se apropriar das demandas em saúde mental existentes no território. Entretanto, os discursos dos profissionais de saúde revelam que a territorialização é realizada apenas pela equipe multidisciplinar da APS, sem a participação dos profissionais do CAPS:

Eu trabalho no CAPS há dois anos e aqui não é feita a territorialização (...), a territorializaçãoérealizada pela atenção básica; eles definem o território de abrangência da regional e nós consideramos a territorialização de lá (Enf. 18).

A territorialização é realizada pelos profissionais da equipe de saúde aqui da atenção básica, enfermeiro, os ACSs, a gente vai pro território pra identificar as 
questões do número de famílias, quais as doenças mais presentes, questão de saneamento e dos problemas mais críticos (ACS-GF 03).

A territorialização é uma ferramenta importantíssima para organização e planejamento das ações em saúde ofertadas para a comunidade. Para os profissionais do CAPS, cujo serviço deve operar conforme a lógica do território (Brasil, 2011), é de extrema importância participar da territorialização, com vistas a reconhecer as demandas em saúde mental e os recursos que permitam a formulação de novas estratégias de cuidado (Prata et al., 2017; Lima e Yasui, 2014). Entretanto, o que se observa são práticas cristalizadas que se restringem aos muros do próprio serviço, que não possibilitam aos profissionais enxergar o território como potencializador de novas formas de cuidar.

Os discursos revelam que os profissionais reconhecem a importância dessa aproximação com o território para a compreensão das necessidades da população. Os ACSs são os profissionais que estão mais próximos da população e convivem no território; embora não estejam capacitados para reconhecerem tais 'sintomas', conseguem identificar a maior parte das demandas em saúde mental na comunidade por meio da visita domiciliar.

(...) Em relação à saúde mental, quem faz essa identificação somos nós [ACS] na visita; quando a gente percebe alguma coisa de errado, nós comunicamos ao enfermeiro (ACS-GF 02).

Geralmente,osagentes[ACS]comunicamagentequandoalgumapessoaestáprecisando de uma assistência em saúde mental (...) eles conseguem identificar essa demanda (Enf. 05).

Os ACSs, se treinados, são atores potencialmente estratégicos para atuar na interface da saúde mental com a atenção básica, pois conseguem identificar as demandas da atenção psicossocial, por meio de sua circulação em dupla jornada pelo território, como morador e trabalhador. Os agentes de saúde são como elos entre os usuários e os serviços de saúde, por residirem na comunidade e estarem mais próximos da realidade do território, podendo, por meio de treinamentos ou capacitações, tornarem-se capazes de identificar as necessidades da população em relação à saúde mental.

Nesse sentido, embora ainda não possuam formação profissional que contemple as especificidades do campo da saúde mental, os ACSs configuram-se como peças importantes para a implementação do cuidado psicossocial com base territorial. Nos serviços investigados, visualizam-se ações territoriais pautadas na visita domiciliar como um instrumento de cuidado extramuros. Essas visitas são realizadas, principalmente, pelos agentes de saúde ACS, mas 
também, pelos enfermeiros e médicos do CAPS, quando o usuário não consegue ir até o serviço, como é possível depreender nas falas a seguir:

A gente faz a visita domiciliar e acompanha a pessoa que faz uso de medicação controlada, a gentevêse tá tomando direitinho, ese perceber alguma coisa errada, a gente informa pro enfermeiro da equipe, ele avalia, e seachar necessário fazuma visita naquela casa ou mesmo marca uma consulta (ACS-GF 04).

Oagente que passa na minha casa sempre pergunta como eu tô, se eu tô tomando a medicação direitinho, mas só isso, acho que eles também não podem fazer muita coisa, né? (Usuário 22).

A gente quando precisa faz uma visita domiciliar, quando o paciente é idoso e não consegue vir até o CAPS, então sempre tem um dia que nós atendemos pela visita (Enf. 17).

Segundo Saffer e Barone (2017), o ACS é um dos principais atores da ESF e suas funções foram se ampliando progressivamente entre os novos campos, dentre os quais, o da saúde mental. Entretanto, as práticas dos ACSs vêm sendo alvo de discussões, pois devido à aproximação da Atenção Básica ao convívio cotidiano dos usuários, abre-se espaço para uma gama de subjetivações e atitudes afetadas pelas políticas de saúde, o que pode se refletir em um aumento, dentre outras possibilidades, de medicalização das comunidades.

O trabalho do ACS insere-se em um campo de disputa, permeado de conflitos, porque ele pertence à comunidade tanto como morador do território quanto como trabalhador da equipe que atende à área em que habita (Saffer e Barone, 2017). Esta condição exige do ACS criatividade para lidar com situações de trabalho desafiadoras, considerando sua inserção na mesma cultura e nas dificuldades das famílias acompanhadas (Samudio et al., 2017).

Estudo de Samudio et al. (2017) mapeou a produção de cuidado em saúde mental que acontece no encontro entre o ACS e o usuário na atenção básica. Os autores revelaram entraves que podem endurecer o cuidado em saúde mental e as possibilidades de se produzirem novas formas de cuidado. Os entraves diziam respeito às condições de trabalho desfavoráveis, uma vez que os agentes comunitários compõem equipes transcendentes no cuidado em saúde e saúde mental. Já as possibilidades produtoras de cuidado circularam ante a abertura dos ACSs ao 'aprender', além da constatação de movimentos desses profissionais favoráveis à construção de novos modos de cuidado em saúde mental, como 'grupos de escuta' e 'pensar em resoluções coletivas'.

Outros estudos mostram a importância do ACS na identificação de demandas de saúde mental em função do trânsito entre a convivência com a comunidade e o equipamento de saúde que referencia. Desse modo, o ACS 
encontra-se numa posição estratégica para esta articulação (Harada e Soares, 2010; Moura e Silva, 2015; Tatmatsu e Araújo, 2016). Para Waidman, Costa e Paiano (2012), os ACSs, por compartilharem o mesmo contexto social e por conhecerem de perto a dinâmica da comunidade, são considerados importantes facilitadores no cuidado à saúde mental.

Destarte, as práticas territoriais são potentes, pois oferecem a possibilidade de produzir cuidado nos espaços da vida cotidiana, com reinserção do usuário ao seio familiar e ao convívio social. Por outro lado, porém, o que se observa nos discursos dos profissionais e usuários é que persiste a valorização de práticas de cuidado dentro do próprio serviço, sem articulação com o território:

Nóstemos vários gruposterapêuticos aquinoCAPS, comoterapeuta ocupacional, psicólogo, enfermeiro, esses grupos funcionam aqui no próprio serviço (...). $\mathrm{Na}$ comunidade, hoje em dia, estamos sem programação (Enf. 17).

Eu já participei de um grupo aqui no CAPS, mas hoje não participo mais. Na minha comunidade, não tem nada não, pelo menos que eu conheça, não (Usuário 12).

Resultados semelhantes aos apresentados foram encontrados em estudo produzido em um município no estado do Paraná; verificou-se que a maioria dos profissionais de saúde desenvolve suas práticas em grupos, com atividades lúdicas que promovem a interação. Entretanto, esses grupos são formados intramuros do serviço de saúde. Nesse sentido, a organização dos processos de trabalho não favorece ao cuidado territorial (Oliveira e Caldana, 2016).

Essa lógica de atuação do CAPS permanece centrada no modelo asilar, visto que promove práticas de cuidados para população intramuros do serviço, sem se preocupar em adentrar no território e considerar o contexto sociocultural, familiar e político dos usuários. Em resposta à essa lógica, a realização do apoio matricial pode oferecer subsídios para que os profissionais da APS se tornem capazes de intervir e conduzir os casos mais simples de saúde mental de sua área de cuidado. Entretanto, no município estudado, essa estratégia não foi priorizada pela gestão nos últimos anos.

Segundo Bezerra e Jorge (2018), os entraves para a implementação e funcionamento do matriciamento pode estar relacionado à falta de sensibilização, de conhecimento técnico e teórico por parte dos gestores, traduzindo-se na resistência dos profissionais em participar do apoio matricial.

Nunes, Guimarães e Sampaio (2016) afirmam que práticas intramuros dos CAPSs se configuram como abordagens reducionistas do usuário, centradas na clínica individual, tratando-o como objeto. Para Leão e Barros (2012), quando o serviço foca a sua atenção em ações exclusivamente centradas na medicação 
ou em atividades intramuros, que não se relacionam com o exterior, inevitavelmente, reproduz a lógica do modo asilar.

Contudo, para ressignificar o território como um espaço de cuidado são necessárias condições que permitam o planejamento e a organização dos serviços e, nos discursos dos profissionais, a ausência dessas práticas de cuidado no território é justificada pela falta de condições de trabalho e segurança no entorno das comunidades, como pode ser aferido em seus relatos:

O grande problema de promover ações na comunidade é a falta de condições de trabalho; se eu quiser ir pra comunidade, tenho que ir no meu carro, correndo perigo de ser assaltada, e isso dificulta muito (Enf. 07).

As ações na comunidade demandam tempo e aqui nós temos um contingente de profissional reduzido, somos sobrecarregados aqui no CAPS (Enf. 23).

A precariedade das condições de trabalho favorece a desresponsabilização do profissional frente aos problemas de saúde dos usuários. Guimarães, Jorge e Assis (2011) afirmam que é preciso melhorar as condições de trabalho, incluindo a aquisição de materiais necessários para o desenvolvimento das atividades.

Ressalta-se, também, a necessidade de democratização das relações de trabalho, mediante transformações na organização dos processos de trabalho, com a finalidade de promover autonomia para os trabalhadores criarem novas opções terapêuticas.

As diretrizes da Política de Saúde Mental definem que a territorialização dos usuários da saúde mental deve ser realizada com o objetivo de inserí-los em um serviço de saúde mental aberto, na família, na comunidade, nos locais onde possam usufruir de todos os direitos que garantam sua cidadania (Brasil, 2013; Guedes e Souza, 2015).

Estudo produzido nos Estados Unidos observou que os cuidados realizados por profissinoais da atenção primária em saúde têm assumido um papel cada vez mais importante no atendimento ambulatorial, tendo em vista a formação de vínculos entre os profissionais e os usuários, o que representa um papel de vital importância no cuidado para esta população (Olfson, 2016).

As visitas domiciliares são instrumentos de trabalho preciosos no cuidado ao paciente em sofrimento psíquico, sendo utilizadas nas mais diferentes formas de acompanhamento dos pacientes. Com elas, é possível estabelecer uma aproximação com a realidade vivida pelos sujeitos e seu contexto familiar, permitindo pensar em soluções que a comunidade possa oferecer (Pereira et al., 2014).

Todavia, a visita domiciliar não pode ser o único instrumento para produzir inovações práticas em saúde mental, e sim, partir dela para repensar novas formas de cuidado. Dentre as atividades práticas de cuidado territoriais 
observadas pelos profissionais, os enfermeiros citaram o grupo de terapia comunitária realizada no território: como pode ser observado nos discursos a seguir:

Aqui a gente faz a terapia comunitária de grupo. Participa a fonoaudióloga, o enfermeiroeoagente [comunitário] de saúde; a gentevaiatéa comunidade,emuma quadra, e organiza o grupo uma vez a cada 15 dias (Enf. 23).

Na nossa unidade, existe um grupo de terapia comunitária que eu mesmo faço, no Lagamar [bairro], com gestantes em situação de violência (Enf. 05).

A terapia comunitária caracteriza-se como proposta terapêutica para a área de saúde mental, com o intuito de se tornar um espaço no qual as pessoas podem ser acolhidas nas suas dores e nos seus sofrimentos, ocasionados por qualquer obstáculo comum do dia a dia, ou mesmo por situações traumáticas, que causaram, ou têm um potencial de causar algum transtorno mental. Tem, em sua base de formação, elementos que valorizam a diversidade cultural, a autonomia, a visão sistêmica, o potencial comunitário e as subjetividades (Arruda, 2011).

Para Souza et al.(2011), a terapia comunitária consegue transformar espaços e construir saberes e, nesses espaços, os participantes têm acesso a uma assistência integral e singular. Nesse contexto, ela gera contribuições no âmbito social e familiar e leva cada usuário a uma nova forma de pensar e agir sobre as dificuldades da vida, melhorando a autoestima, a resiliência, como também sua estabilidade emocional, com redução da ansiedade e, principalmente, do estresse.

Em meio ao contexto apresentado, evidencia-se a necessidade latente de os serviços ocuparem os espaços dos bairros, não só com atividades informativas, mas com ações que possam ampliar e melhorar sua prática clínica consoantes com as especificidades dos contextos territoriais nos quais estes usuários vivem. A rede de atenção psicossocial deve retirar o serviço da invisibilidade, ampliando a teia de diálogos com a vizinhança e contribuindo para a busca da equidade, por meio da territorialidade (Santos e Nunes, 2011). Como afirmam Covelo e Badaró-Mareira (2015), além dos serviços, é importante lembrar que existem outros espaços que podem potencializar o cuidado em saúde mental, como o território. Pensar na territorialidade é pensar em uma rede para fora dos serviços, permitindo reconhecer as singularidades e complexidades de cada sujeito, visto que cada um possui um território, carregado de história, relações de poder, relações sociais, formas de organização e economia.

O território é autônomo e possui movimento, marcado por transformações advindas dessa organização histórica e social das populações. Perceber e incorporar este território que transcende o físico é essencial para o avanço na constituição e implementação de políticas públicas em saúde mental (Silva e Pinho, 2015). Nesse sentido, o trabalho no território dimensiona a construção de novas relações 
sociais entre todos os atores envolvidos, produzindo modificações nos contextos nos quais ainda se faz presente uma cultura de exclusão (Leão e Barros, 2012).

Nesse contexto, o território no qual o sujeito vive tem se mostrado uma ferramenta importante, que não deve ser desconsiderada durante o tratamento, devendo estar integrada aos espaços institucionais. Com efeito, a rede territorial é palco das relações sociais que constituem um sujeito e nela se produz e reproduz um conjunto de referências socioculturais, políticas e econômicas, que qualifica seu cotidiano e sua inserção no mundo (Kemper et al., 2015). Nesse sentido, as práticas territoriais valorizam o usuário como ser social, cidadão, autônomo, único, com capacidade de melhorar sua saúde. Destarte, as práticas devem acontecer de dentro para fora dos serviços, ou seja, no território onde os usuários vivem, já que o modus operandi dessa proposta é a relação; assim, pode-se dizer que o profissional se ocupa, permanentemente, da negociação com pessoas ou recursos do território (Dutra e Oliveira, 2015).

Essa presença viva no território implica, necessariamente, o envolvimento de todos os atores comprometidos no cuidado, isto é, a equipe multiprofissional, a família, a comunidade em geral; enfim, de todos que, de alguma forma, façam parte daquele ambiente (Brasil, 2015).

Em síntese, os dados obtidos por este estudo possibilitam compreender a interface entre o que é preconizado pela reforma psiquiátrica e as dificuldades e fragilidades vivenciadas no cotidiano dos profissionais e usuários, possibilitando aos gestores e profissionais ressignificarem as práticas de saúde para implementação de ações individuais e coletivas que permitam alcançar a transversalidade do cuidado.

Como limitações deste estudo, destaca-se a ausência da participação dos gestores, pois a visão desse grupo de profissionais poderia aprofundar a compreensão do processo de cuidado em saúde mental. Constata-se a necessidade de ampliar e identificar as ações preconizadas pela gestão, suas dificuldades e potencialidades para compreensão geral do fenômeno. No entanto, essas limitações não comprometeram a qualidade dos dados obtidos, podendo servir de estímulo para novos estudos.

\section{Considerações finais}

As práticas de cuidado em saúde mental ainda estão pautadas em ações realizadas no próprio serviço e na medicalização do sujeito para tratar os sintomas da doença mental.Tais práticas desconsideram suas especificidades e singularidadesereproduzem umcuidadointramurosqueseconfiguracomomodeloasilar. Dessemodo, perdema potência detransformaçãodas práticas desaúdemental, divergindo do que propõe a reforma psiquiátrica. O panorama da saúde mental urge pela transformação de um modelo que privilegie a revisão epistemológica e a reflexão dos profissionais de saúde, como forma de consolidar novas ações. 
Nesse aspecto, é importante refletir e repensar a criação ou a intensificação de novas práticas de cuidado com bases territoriais que permitam atender às necessidades de saúde dos usuários e considerem o território como um espaço adequado e potente para estas ações de cuidado. Ademais, desenvolver estratégias para capacitar a formação de trabalhadores que atuam na transversalidade da saúde mental, à exemplo dos ACSs, pode ampliar a resolubilidade dos problemas nesse campo e na atenção básica.

Diante do exposto, torna-se um desafio introduzir práticas de cuidados singulares e coletivas capazes de promover uma assistência em saúde mental de qualidade que vai além do saber científico, abrangendo o estabelecimento de relações sólidas com os usuários e a comunidade, com intuito de satisfazer as reais necessidades existentes.

\section{PRODUCIÓN DEL CUIDADO EN SALUD MENTAL: PRÁCTICAS TERRITORIALES EN LA RED PSICOSOCIAL}

Resumen Se trata de un estudio cualitativo que analizó prácticas de cuidado territoriales en salud mental realizadas por enfermeros, agentes comunitarios de salud y usuarios de los centros de atención psicosocial y de la atención básica. Han sido realizadas entrevistas, grupos focales y observación libre con 60 participantes de la ciudad de Fortaleza, estado de Ceará, Brasil, en 2017. El análisis de las informaciones estuvo basado en la hermenéutica-dialéctica ante un ejercicio interpretativo crítico y reflexivo. Los resultados evidenciaron que el proceso de territorialización es realizado por el equipo multidisciplinario de la Estrategia Salud de la Familia, sin la participación de los profesionales del centro de atención psicosocial. Aunque ellos a veces realicen prácticas comunitarias, persiste la valoración de acciones dentro del propio servicio y en la medicalización del sufrimiento psíquico, sin considerar las singularidades de los sujetos, y sin articulación con los servicios de la atención básica. Los agentes comunitarios de salud, si entrenados, son actores potencialmente estratégicos para actuar en la interconexión de la salud mental con la atención básica. De hecho, el panorama de la salud mental urge por la transformación de un modelo que privilegie la reflexión de nuevas acciones en múltiples dimensiones, con énfasis en la articulación de los servicios y en la capacitación de los trabajadores que actúan en ese ámbito.

Palabras clave asistencia a la salud; atención primaria de salud; salud mental; servicios de salud mental; agentes comunitarios de salud.

\section{Colaboradores}

Daniella Barbosa Campos realizou todas as etapas de construção da pesquisa e elaboração do artigo científico. Indara Cavalcante Bezerra participou da concepção do artigo, delineamento teórico e metodológico do texto, da escrita, revisão crítica e aprovação final do manuscrito. Maria Salete Bessa Jorge participou da definição do objeto, construção do artigo e organização final.

\section{Financiamento}

Daniella Barbosa Campos recebeu apoio da Coordenação de Aperfeiçoamento de Pessoal de Nível Superior (Bolsa de Mestrado). 


\section{Referências}

ARDILA-GÓMEZ, Sara et al. Social perceptions about community life with people with mental illness: study of a discharge program in Buenos Aires Province, Argentina. Community Mental Health Journal, [s.l.], v. 51, n. 1, p. 103-110, jan. 2015.

ARRUDA, Amália G. Saúde mental na comunidade: a terapia comunitária como dispositivo de cuidado. Dissertação (Programa de Mestrado Acadêmico em Saúde Pública). Universidade Estadual do Ceará, Centro de Ciências da Saúde. Fortaleza, 2011.

ASSIS, Marluce M. A.; JORGE, Maria S. B. Métodos de análise em pesquisa qualitativa. In: SANTANA, Judith S. S.; NASCIMENTO, Maria A. A. (Orgs.). Pesquisa: métodos e técnicas de conhecimento da realidade social. Feira de Santana: UEFS Editora, 2010. p.139-159.

BEZERRA, Indara C.; JORGE, Maria S. B. “Não temos nenhuma equipe matricial funcionando $[\ldots . .]^{\prime \prime}$ : matriciamento como dispositivo de cuidado em saúde mental. In: JORGE, Maria; CARVALHO, Maria; COSTA, Lourdes (Org.). Dispositivo do cuidado no contexto do matriciamento em saúde mental na atenção primária: articulações entre saberes e práticas. Fortaleza: EdUECE, 2018. p. 178-190.

BRASIL. Conselho Nacional de Saúde. Resolução $n^{\circ} 466,2012$. Diretrizes e Normas regulamentadoras de pesquisa envolvendo seres humanos. Diário Oficial da União, Brasília, DF, 12 dez. 2012. Seção 1 p. 59.

BRASIL. Ministério da Saúde. Portaria ${ }^{\circ}$ 3.088, de 23 de dezembro de 2011. Institui a Rede de Atenção Psicossocial para pessoas com sofrimento ou transtorno mental e com necessidades decorrentes do uso de crack, álcool e outras drogas, no âmbito do Sistema Único de Saúde. Diário Oficial da União, Brasília, DF, 26 dez. 2011. Seção 1, p. 230-2.

BRASIL. Ministério da Saúde. Saúde Mental. Curso de aperfeiçoamento módulo 5 - Dispositivos de atenção psicossocial. Ministério da Saúde: Brasília, 2015.
BRASIL. Ministério da Saúde. Secretaria de Atenção à Saúde. Departamento de Atenção Básica. Saúde mental. Ministério da Saúde, Secretaria de Atenção à Saúde, Departamento de Atenção Básica, Departamento de Ações Programáticas Estratégicas. Brasília: Ministério da Saúde, 2013.

CASTRO, Leonardo M.; MAXTA, Bruno S. B. Práticas territoriais de cuidado em saúde mental: experiências de um centro de atenção psicossocial no município do Rio de Janeiro. SMAD, Revista Eletrônica Saúde Mental Álcool e Drogas, Ribeirão Preto, v. 6, n. 1, p. 1-11, 2010.

COVELO, Bárbara S. R.; BADARÓ-MOREIRA, Maria I. Laços entre família e serviços de Saúde Mental: a participação dos familiares no cuidado do sofrimento psíquico. Interface: Comunicação, Saúde e Educação, Botucatu, v. 19, n. 55, p. 1133-1144, dez. 2015.

DIEZ-CANSECO, Francisco et al. Integration of a technology-based mental health screening program into routine practices of primary health care services in Peru (The Allillanchu Project): development and implementation. Journal of Medical Internet Research, Toronto, v. 20, n. 3, e100, 2018.

DUTRA, Virginia F. D.; OLIVEIRA, Rosane M. P. Revisão integrativa: as práticas territoriais de cuidado em saúde mental. Aquichán, Bogotá, v. 15, n. 4, p. 529-540, out. 2015.

FERNANDES, Amanda D. S. A.; MATSUKURA, Thelma S.; LOURENÇO, Mariana S. G. Práticas de cuidado em saúde mental na Atenção Básica: identificando pesquisas no contexto brasileiro. Cadernos Brasileiros de Terapia Ocupacional, São Carlos, v. 26, n. 4, p. 904-914, dez. 2018.

FORTALEZA (Cidade). Secretaria Municipal da Saúde. Plano municipal de saúde de Fortaleza: 2018-2021. Fortaleza: Secretaria Municipal da Saúde, 2017. 167 f.

GUEDES, Patrícia F. M.; SOUZA, Maria C. R. F. Cartografias de exclusão e inclusão 
de pessoas com sofrimento mental nos processos de territorialização da Política Nacional de Saúde Mental. Estudos e Pesquisas em Psicologia, Rio de Janeiro, v. 15, n. 1, p. 40-57, abr. 2015.

GUIMARÃES, José M. X. Inovação e gestão em serviços de saúde mental: incorporação de tecnologias e reinvenção no cotidiano dos Centros de Atenção Psicossocial. 2012. 286f. Tese (Doutorado em Saúde Coletiva)Universidade Estadual do Ceará, Centro de Ciências da Saúde, Programa de Doutorado em Saúde Coletiva, Fortaleza, 2012.

GUIMARÃES, José M. X.; JORGE, Maria S. B.; ASSIS, Marluce M. A. (In)satisfação com o trabalho em saúde mental: um estudo em centros de atenção psicossocial. Ciência \& Saúde Coletiva, Rio de Janeiro, v. 16, n. 4, p. 2145-2154, abr. 2011.

HARADA, Olívia L.; SOARES, Marcos H. A percepção do agente comunitário de saúde para identificar a depressão. SMAD, Revista Eletrônica Saúde Mental Álcool e Drogas, Ribeirão Preto, v. 6, n. 2, p. 315336, ago. 2010.

KEMPER, Maria L. C. et al. Integralidade e redes de cuidado: uma experiência do PETSaúde/Rede de Atenção Psicossocial. Interface: Comunicação, Saúde e Educação, Botucatu, v. 19, supl. 1, p. 995-1003, 2015.

KILBOURNE, Amy M. et al. Measuring and improving the quality of mental health care: a global perspective. World Psychiatry, [s.l.], v. 17, n. 1, p. 30-38, 2018.

LANCETTI, Antonio; AMARANTE, Paulo. Saúde mental e saúde coletiva. In: CAMPOS, Gastão W. S. et al. (Org.). Tratado de Saúde Coletiva. Rio de Janeiro: HUCITEC; Fiocruz, 2006. p. 615-634.

LEÃO, Adriana; BARROS, Sônia. Território e serviço comunitário de saúde mental: as concepções presentes nos discursos dos atores do processo da reforma psiquiátrica brasileira. Saúde e Sociedade, São Paulo, v. 21, n. 3, p. 572-586, set. 2012.
LIMA, Elizabeth M. F.; YASUI, Silvio. Territórios e sentidos: espaço, cultura, subjetividade e cuidado na atenção psicossocial. Saúde em Debate, Rio de Janeiro. v. 38, n. 102, p. 593606, 2014.

MACIEL, Marcelo A. Reforma psiquiátrica: as experiências francesa e italiana. Cadernos de Saúde Pública, Rio de Janeiro, v. 26, n. 9, p. 1863-1864, set. 2010.

MARCOLINO, Taís Q.; FANTINATTI, Eliane N.; GOZZI, Alana P. N. F. Comunidade de prática e cuidado em saúde mental: uma revisão sistemática. Trabalho, Educação e Saúde, Rio de Janeiro, v. 16, n. 2, p. 643-658, ago. 2018.

MINAYO, Maria C. O desafio do conhecimento: pesquisa qualitativa em saúde. 11. ed. São Paulo: Editora Hucitec, 2008.

MINAYO, Maria C. Pesquisa social: teoria, método e criatividade. 14. ed. Petrópolis: Editora Vozes, 1999.

MINAYO, Maria C. Pesquisa Social: teoria, método e criatividade. 29. ed. Petrópolis: Editora Vozes, 2010.

MOURA, Raul F. S.; SILVA, Carlos R. C. Saúde mental na Atenção Básica: sentidos atribuídos pelos agentes comunitários de saúde. Psicologia: Ciência e Profissão, Brasília, v. 35, n. 1, p. 199-210, mar. 2015.

NUNES, Jeanine M. S.; GUIMARÃES, José M. X.; SAMPAIO, José J. C. A produção do cuidado em saúde mental: avanços e desafios à implantação do modelo de atenção psicossocial territorial. Physis: Revista de Saúde Coletiva, Rio de Janeiro, v. 26, n. 4, p. 12131232, out. 2016.

OLFSON, Mark. The rise of Primary Care physicians in the Provision of US mental health care. Journal of Health Politics, Policy and Law, Chapel Hill, v. 41, n. 4, p. 559-583, 2016.

OLIVEIRA, Thaís T. S. S.; CALDANA, Regina H. L. Psicologia e práticas psicossociais: narrativas e concepções de psicólogos de centros de atenção psicossocial. Revista Estudos Inter- 
disciplinares em Psicologia, Londrina, v. 7, n. 2, p. 22-44, dez. 2016.

ONOCKO-CAMPOS, Rosana T. et al. Avaliação da rede de centros de atenção psicossocial: entre a saúde coletiva e a saúde mental. Revista de Saúde Pública, São Paulo, v. 43, supl. 1, p. 16-22, ago. 2009.

PEREIRA, Sandra S. et al. Visita domiciliar aos pacientes portadores de transtorno mental: ampliando as opções terapêuticas possíveis em um serviço ambulatorial. Saúde \& Transformação Social, Florianópolis, v. 5, n. 1, p. 91-95, 2014.

PRATA, Nina I. S. S. et al. Saúde mental e Atenção Básica: território, violência e o desafio das abordagens psicossociais. Trabalho, Educação e Saúde, Rio de Janeiro. v. 15, n. 1, p. 33-53, 2017.

SAFFER, Denis A.; BARONE, Luciana R. Em busca do comum: o cuidado do agente comunitário de saúde em Saúde Mental. Physis: Revista de Saúde Coletiva, Rio de Janeiro. v. 27, n. 03, p. 813-833, 2017.

SAMPAIO, José J. C.; GUIMARÃES, José M. X.; ABREU, Luciana M. Supervisão clínico-institucional e a organização da atenção psicossocial no Ceará. São Paulo: Editora HUCITEC, 2010.

SAMUDIO, Jania L. P. et al. Cartografia do cuidado em saúde mental no encontro entre agente comunitário de saúde e usuário. Physis: Revista de Saúde Coletiva, Rio de Janeiro. v. 27, n. 2, p. 277-295, 2017.

SANTOS, Marcos R. P. S.; NUNES, Mônica O. Território e saúde mental: um estudo sobre a experiência de usuários de um Centro de Atenção Psicossocial, Salvador, Bahia, Brasil. Interface: Comunicação, Saúde e Educação, Botucatu, v. 15, n. 38, p. 715-726, set. 2011.
SILVA, Aline B.; PINHO, Leandro B. Território e saúde mental: contribuições conceituais da geografia para o campo psicossocial. Revista Enfermagem UERJ, Rio de Janeiro, v. 23, n. 3, p. 420-424, 2015.

SOUZA, Gleci M. L. et al. A contribuição da terapia comunitária no processo saúde-doença. Cogitare Enfermagem, Curitiba, v. 16, n. 4, p. 682-688, 2011.

SOUZA, Miriam C.; AFONSO, Maria L. M. Saberes e práticas de enfermeiros na saúde mental: desafios diante da Reforma Psiquiátrica. Gerais: Revista Interinstitucional de Psicologia, Juiz de Fora, v. 8, n. 2, p. 332-347, dez. 2015.

SOUZA, Tadeu P.; CARVALHO, Sérgio R. Apoio territorial e equipe multirreferencial: cartografias do encontro entre $o$ apoio institucional e a redução de danos nas ruas e redes de Campinas, SP, Brasil. Interface: Comunicação, Saúde e Educação, Botucatu, v. 18, supl. 1, p. 945-956, 2014.

TATMATSU, Daniely B.; ARAÚJO, Ana C. C. Atenção primária e saúde mental: contribuições e potencialidades do apoio matricial. Mudanças: Psicologia da Saúde, São Bernardo do Campo, v. 24, n. 2, p. 71-79, 2016.

TEIXEIRA, Melissa R.; COUTO, Maria C. V.; DELGADO, Pedro G. G. Repercussões do processo de reestruturação dos serviços de saúde mental para crianças e adolescentes na cidade de Campinas, São Paulo (2006-2011). Estudos de Psicologia, Campinas, v. 32, n. 4, p. 695703, dez. 2015.

WAIDMAN, Maria A. P.; COSTA, Bruna; PAIANO, Marcelle. Percepções e atuação do Agente Comunitário de Saúde em saúde mental. Revista da Escola de Enfermagem da USP, São Paulo, v. 46, n. 5, p. 1170-1177, out. 2012. 